\title{
Desigualdades sociales desde el enfoque de la complejidad. Integrando disciplinas para pensar nuestra realidad
}

\section{Social inequalities from the perspective of complexity. Integrating disciplines to think our reality}

\begin{abstract}
ARTÍCULO
\section{Bárbara Altschuler}

Universidad Nacional de Quilmes. Observatorio del Sur de la Economía Social y Solidaria, Argentina. baltschuler@unq.edu.ar

Recibido: junio del 2016

\section{Resumen}

La desigualdad social constituye un problema crucial de las sociedades latinoamericanas y un problema teórico debatido ampliamente por las ciencias sociales. A pesar de los avances en la conceptualización de la desigualdad como un fenómeno complejo, su abordaje desde este enfoque no ha sido resuelto satisfactoriamente. Las dificultades, vinculadas con la especialización desarticulada en que se desarrollaron las disciplinas sociales, se manifiestan a su vez en tensiones y dualismos que las atraviesan. Planteamos aquí algunos conceptos clave que permitirían avanzar en la articulación entre disciplinas, e indagamos en los aportes y desafíos que representa el paradigma complejo para pensar esta realidad.
\end{abstract}

Palabras claves: desigualdad social; ciencias sociales; complejidad; articulación interdisciplinaria; figuración social.

\section{Abstract}

Social inequality is a crucial problem of Latin American societies and a widely debated theoretical issue in social sciences. Despite the improvements in the conceptualization of inequality as a complex phenomenon, a satisfactory approach from this point of view has not been reached yet. The difficulties, related to the disconnected specialization in which the social disciplines were developed, manifest themselves in the tensions and dualities crossing social sciences. We propose some key concepts which would potentially help us to advance in the relationship between disciplines, and we explore the contributions and challenges that this complex paradigm for thinking about this reality represents. 
Key words: social inequality; social sciences; complexity; interdisciplinary articulation; social figuration. 


\section{Introducción}

La desigualdad social constituye en la actualidad un problema crucial de las sociedades latinoamericanas, afectadas en su mayoría por la implementación de modelos neoliberales en el último cuarto de siglo. Estos vinieron a agudizar las desigualdades históricas de diverso tipo que ya caracterizaban a estas sociedades. Si bien algunos países de la región implementaron políticas posneoliberales en la última década (tales como Argentina, Brasil, Bolivia o Ecuador) con diversos alcances redistributivos, de inclusión social y disminución de la pobreza, en términos generales los índices de desigualdad no se modificaron sustantivamente (Grimson, et al, 2008). En la mayoría, el agravamiento de los fenómenos de pobreza, desempleo, precariedad laboral y exclusión, concomitantes con procesos de concentración y extranjerización de la riqueza en sectores clave de la economía y en el marco de la globalización económica y financiera, han llevado, según los casos, a un sostenimiento y/o profundización de las desigualdades sociales.

Asimismo, la desigualdad económica y social de las sociedades modernas ha sido un problema teórico abordado y debatido ampliamente desde las ciencias sociales, tanto por autores clásicos como Weber y Marx, como por autores contemporáneos como Bourdieu (1979 y 1984), Rosanvallón (1995), Castel (1995), Tilly (2000), Svampa (2005), Wieviorka, (2006), Reygadas (2008), Grimson (2008) y Kessler (2014), entre muchos otros. Su estudio ha tenido una creciente relevancia analítica en los últimos años, debido a la constatación de su persistencia y/o incremento, incluso en períodos de crecimiento económico. También, debido a la mayor visibilidad que han adquirido en la arena política y cultural otros tipos de desigualdades, como las étnicas, de género, sexuales o socio-ambientales. Ambas cuestiones han contribuido a una revitalización del debate sobre la desigualdad, reconociendo a la misma como un fenómeno multidimensional y multideterminado (Reygadas, 2008).

Sin embargo, a pesar de la existencia de esfuerzos significativos en el avance de las diversas disciplinas sociales para el estudio interdisciplinario de realidades complejas, consideramos que éstos no constituyen avances sistemáticos ni generalizados, persistiendo sobre el estudio de las desigualdades, en términos generales, enfoques unidimensionales, ahistóricos y/o no relacionales. En este marco, nos proponemos analizar algunas dificultades de las ciencias sociales para articular saberes disciplinares, e indagar en los aportes que el paradigma de la complejidad nos brinda para el estudio de las desigualdades sociales. El objetivo principal del trabajo es aportar a un estudio más integral de las desigualdades sociales, analizando a la misma en tanto fenómenos sociales complejos.

Planteamos para ello, en primer lugar, las dificultades encontradas en los estudios sobre desigualdades sociales e indagamos dos dualidades o tensiones al interior de las ciencias sociales vinculadas a la temática propuesta: la tensión entre individuo- sociedad y la tensión entre igualdad- diferencia. Sostenemos que éstas resultan del pensamiento disyuntivo $y$ 
simplificador que caracterizó el desarrollo de las ciencias sociales (Morín, 1977 y 1994) y reflejan el aislamiento en que las mismas se desarrollaron desde sus orígenes (Schorske, 1981). A partir de ello, proponemos, en segundo lugar, algunos conceptos y enfoques que aportan a un estudio más integral de los fenómenos sociales en general y de las desigualdades sociales en particular y se tienen en cuenta cuestiones teóricas y metodológicas para un enfoque complejo de las desigualdades sociales. Por último, se recuperan algunos aportes que el paradigma de la complejidad puede realizar para el estudio de la temática.

Rolando García define a los sistemas complejos como aquellos que "están constituidos por elementos heterogéneos en interacción" (García, 2007, p. 32). Edgar Morín señala que lo complejo es lo multidimensional, aquello que "reúne en sí orden, desorden, organización, y, en el seno de la organización lo uno y lo diverso" (Morín, 1994, p. 24), aunque lo complejo no debe confundirse con la completud, que resulta, por definición, inalcanzable. Así, cuando aludimos a "lo complejo" nos referimos a lo multidimensional dentro de cierta unidad y a las relaciones recíprocas entre múltiples dimensiones, o podemos decir también, a las relaciones entre las partes y el todo. Se trata, claro, de un todo estructurado de manera asimétrica y diversa, configurado socio-históricamente y atravesado por relaciones de poder.

Coincidiendo con el espíritu desafiante y entusiasta de Edgar Morín, nos internamos en este camino en tanto "aventura intelectual" que no constituye un proceso acabado sino, muy por el contrario, "un camino que se hace andando" (Morín, 1994); una trayectoria que resulta única, dado que siempre implica, como nos propone el enfoque complejo, a la propia subjetividad y contexto del investigador. Recuperamos así un largo proceso de trabajo realizado desde mis estudios de Doctorado en Ciencias Sociales, en cuya tesis me interrogo por las desigualdades sociales en una configuración socio-histórica particular, intentando avanzar desde una perspectiva compleja ${ }^{1}$.

\section{Tensiones y dificultades de las ciencias sociales para un abordaje complejo}

\subsection{Limitaciones de los estudios convencionales sobre desigualdad social}

Actualmente resulta casi un lugar común decir que América Latina es la región más desigual del mundo. Sin embargo, son muchas las dificultades y restricciones que encontramos en los abordajes de la cuestión para considerar la problemática en toda su profundidad y complejidad.

\footnotetext{
${ }^{1}$ En dicha tesis estudio las modalidades y dimensiones de la desigualdad social en una configuración socio-histórica particular (la reestructuración del sector vitivinícola de la provincia de Mendoza, Argentina, en las últimas décadas), así como los mecanismos de poder, legitimación y cuestionamiento de tales desigualdades (Altschuler, 2016, IDES- UNGS). Dirigida por el Dr. Alejandro Grimson y co-dirigida por la Dra. Patricia Collado. Agradecemos también en este marco a los evaluadores anónimos del presente artículo que realizaron interesantes observaciones que permitieron mejorar la claridad y precisión del mismo.
} 
Buena parte de los estudios siguen siendo predominantemente económicos y cuantitativos, o bien socio-económicos pero de carácter descriptivo y estático. Nos referimos a una gran cantidad de trabajos realizados en base a indicadores de nivel de ingresos para determinar Línea de Pobreza o Indigencia (LP y LI), índice de Necesidades Básicas Insatisfechas (NBI), o índice de Gini (que mide desigualdad de ingresos). Claramente estas mediciones resultan útiles, pero insuficientes para la comprensión del fenómeno, en tanto no abordan una mirada multidimensional y dinámica (histórica) de los procesos.

Asimismo, si bien las desigualdades aluden inevitablemente a fenómenos relacionales entre grupos sociales y clases, muchos estudios se retrotraen a categorías no relacionales como pobreza, vulnerabilidad social o exclusión, perdiendo de vista la dimensión relacional y de la totalidad, fundamental para comprender el problema en sus causas y complejidad.

En tercer lugar, pocos estudios aluden a las diversas dimensiones de las desigualdades sociales que, como ya señalamos, no solamente son socioeconómicas sino también -y muy fuertemente en América Latina- desigualdades étnico-raciales (ya que coexisten en subordinación múltiples y diversos grupos étnicos, lenguas, prácticas y sentidos culturales); de género (dada la persistencia de fuertes estructuras patriarcales); políticas (que se verifican en importantes asimetrías de poder y participación en la vida pública); territoriales y ambientales (disputas por el acceso a la tierra y los recursos naturales, diferenciales en las oportunidades de desarrollo de acuerdo a la región, etc.); y, en articulación con todas las anteriores, desigualdades simbólicas (en cuanto a brechas de status, reconocimiento y valoración social), por señalar las principales.

En este marco, nos interesa indagar sobre algunas cuestiones que entendemos tensionan a las ciencias sociales y dificultan avanzar en un trabajo interdisciplinario para el abordaje de problemas complejos, como es el análisis las desigualdades sociales.

\subsection{Sociología y Antropología. Dualismo entre igualdad y diferencia}

La sociología, que se consolida como ciencia entre 1830 y 1895 , surge como respuesta a la crisis de los vínculos sociales que significó la revolución industrial en el siglo $\mathrm{XIX}^{2}$. Su origen se vincula, como señala De Ipola (2005), con la pregunta por el lazo social: ¿por qué hay sociedad, el ser-con-los-otros? Es decir, se interroga por las formas de integración social, pero también y casi al mismo tiempo, se formula la pregunta contraria ¿por qué hay lo anti-social, lo a-social, la atomización, el conflicto, la guerra? De aquí en más, la tensión entre integración y

\footnotetext{
2 Emile Durkheim, considerado uno de los "padres de la sociología", le preocupaba que la sociabilidad pre- capitalista no hubiera sido reemplazada por nuevas formas (orgánicas) de lazo social; lo que lo inquietaba, era la atomización social y el auge de los conflictos en la nueva sociedad industrial. Por otra parte, su insistencia estaba puesta en marcar la autonomía e institucionalización de la sociología como ciencia, lo cual se refleja en su frase ya clásica "Lo social se explica por lo social" (Durkheim, 1895).
} 
conflicto, y vinculada a ésta, la tensión entre igualdad y diferencia, recorrerá al conjunto de las ciencias sociales, y a la sociología en particular ${ }^{3}$.

En este marco, la sociología se especializó en estudios sobre la conformación de la estructura social y las clases sociales -en los enfoques de orientación marxista- o bien estudios sobre estratificación social -en el caso de enfoques weberianos. Sin embargo, los estudios clásicos se vieron atravesados desde la segunda mitad del siglo XX por una serie de debates teóricos y procesos sociales que tuvieron como resultado el relegamiento de la perspectiva de clases y el análisis de la estructura social, especialmente desde los años 1980 y 90 . Entre los procesos sociales que hacen al contexto socio-político de la época podemos mencionar: el fin de la "bipolaridad" mundial y la "guerra fría" así como la posterior "neoliberalización" del mundo y la "globalización"; la crisis del fordismo y del Estado de Bienestar y la consiguiente fragmentación y heterogeneización social al interior de las categorías de clase (típicamente pensadas desde el marxismo por la oposición capital-trabajo). Al mismo tiempo, el surgimiento de "nuevos movimientos sociales" desde los años 70 y 80 , como los movimientos feministas, por la diversidad sexual, indígenas y de "minorías" étnicas o raciales, que ayudaron a instalar en la agenda política y en la teoría social nuevos clivajes de desigualdad como los de género y etnia. Dichos procesos tuvieron como resultado el descentramiento de la noción de clase como categoría privilegiada para el análisis del conflicto y la desigualdad social, ampliando las miradas sobre otros procesos y dimensiones.

En el plano teórico, las reconceptualizaciones y aportes de sociólogos como Pierre Bourdieu (1984 y 1979) o Anthony Guiddens (1979) se orientaron a desreificar la estructura y dinámica de clases, tal como eran presentadas por las corrientes más ortodoxas del marxismo. Si bien estos autores reivindicaron los principales hallazgos de Karl Marx, intentaron también articularlos con aportes fundamentales de Max Weber y Emile Durkheim, considerados autores "clásicos de la sociología". Algunos de estos aportes -de especial interés para nuestro tema- se orientaron al cuestionamiento de la determinación unidireccional de la dimensión económica, al análisis de las funciones sociales de los bienes y las prácticas simbólicas, y al estudio de las formas de legitimación de un determinado orden social. Tales contribuciones ayudaron a comprender no sólo los mecanismos de producción de las estructuras, jerarquías y desigualdades sociales, sino también los de reproducción y sostenimiento de las mismas.

Estos "cruces de caminos" o "fertilizaciones mutuas", como diría Morin (1994, p. 12), dieron lugar a análisis enriquecidos, permitiendo superar algunos de los dualismos señalados, como la división entre "lo objetivo" y "lo subjetivo" y entre "lo material" y "lo simbólico" en el análisis de la estructura y la desigualdad social. No sólo lograron articular productivamente -o sea, en un

\footnotetext{
${ }^{3}$ Conviene de entrada señalar que diferencia y desigualdad son conceptos emparentados pero no sinónimos. Mientras que las diferencias (entre individuos, grupos, sociedades) son infinitas y no implican necesariamente una jerarquía o valoración, la desigualdad es un concepto normativo, que implica determinadas definiciones morales y valorativas, tanto por parte del investigador como de los actores sociales, remitiendo a contextos históricos, sociales, políticos y culturales específicos.
} 
sentido enriquecedor y no reduccionista- trayectorias teóricas que parecían irreconciliables dentro de la sociología (como la marxista y la weberiana) sino que además implicaron aperturas y demandas analíticas hacia otras disciplinas, como la antropología y la psicología.

Respecto de la antropología -y su metodología fundamental, la etnografía- su articulación se hizo necesaria para poner en foco procesos micro dentro de las relaciones sociales, en oposición a los macro estudios que caracterizaban a la sociología. Para explicar el interior del mundo social se hizo necesario recurrir a la indagación de las visiones y categorías nativas que construyen y utilizan los actores sociales, las formas de construcción de sentido por parte de estos en la vida cotidiana, los modos de significación siempre situados (en lo histórico y social) de normas, pautas y valores sociales, la performatividad de los bienes y prácticas simbólicas; en síntesis, las formas de mediación cultural que operan en los procesos sociales.

Sin embargo, como señalan Grimson y Seman (2005), la noción de cultura -objeto de estudio privilegiado de la antropología- se encontraría en la actualidad en una situación "paradojal": en el mismo momento en que el conjunto de las ciencias sociales reconoce la importancia de la misma "en un sentido antropológico", la antropología se encuentra en un fuerte debate sobre su definición y utilidad conceptual ${ }^{4}$. Ello se enmarca en un proceso de "autocrítica" y reconceptualización del campo antropológico, en un contexto intelectual marcado desde los años 70 y 80 por las teorías posmodernas, postestructurales, deconstructivas y transdisciplinares que, en términos generales, implican análisis más complejos de la vida social. En este marco, los debates sobre el concepto de cultura se orientaron en una serie de sentidos: a) tendieron a deconstruir las posturas esencialistas, estáticas, mecanicistas y deterministas, tanto de cultura como de identidad (Hall, 2003; Brubaker y Cooper, 2002); b) particularmente en un período como el actual, de creciente "interculturalidad" (Canclini, 2004), se inclinaron a pensar que las culturas no estarían orgánicamente separadas unas de otras y no serían un todo coherente, ni pertenecerían necesariamente a un mismo territorio (Grimson y Seman, 2005); c) a partir del análisis de Barth (1976) sobre "los grupos étnicos y sus fronteras", los trabajos sobre cultura e identidad se orientaron hacia el estudio de las fronteras entre culturas o grupos sociales y al reconocimiento de que sus límites son más o menos borrosos, porosos y asimétricos según los casos (Grimson, 2007; Lamont y Molnár, 2002); y d) en oposición a los llamados "estudios culturales", se planteó la necesidad de reintroducir en el análisis de la cultura las variables históricas y del poder (Ortner, 2005), lo cual implica que lo cultural debe entrelazarse con el análisis de los procesos sociales y políticos, las prácticas y la subjetividad (Ortner, 2005; Williams, 1977).

En síntesis, la antropología crítica tendió al estudio de las diferencias culturales y no de las esencias; al reconocimiento de las disputas sociales por la construcción de sentidos y valores socioculturales; al análisis de las fronteras y mixturas más que de los elementos fijos y estáticos.

\footnotetext{
${ }^{4}$ El sentido antropológico alude a la noción descriptiva y no normativa de las culturas y grupos sociales, cuestión puesta de relevancia especialmente a partir de los aportes de Francis Boas (Cuche, D., 1996).
} 
De este modo, logró problematizar la tensión dual entre igualdad y diferencia y llevó a reconocer la existencia de una relación dialéctica entre ambas, así como también a usar el concepto de cultura para reconocer y problematizar la diversidad, tanto en el tiempo como en el espacio.

Entonces, ¿qué significa la mediación cultural de los procesos y fenómenos sociales y qué vinculación tiene esto con las desigualdades sociales, su cuestionamiento o legitimidad? Dicha mediación implica comprender que existen mecanismos socioculturales a través de los cuales se construye colectivamente el sentido común y los consensos sobre un determinado orden social. Tales construcciones históricas resultan clave para analizar de qué modo y en qué medida las sociedades $-\mathrm{y}$ sus grupos sociales- consideran justo, injusto, aceptable o inaceptable diversos tipos y niveles de desigualdad social. Constituye así una cuestión clave para distinguir la delgada línea, siempre disputada, entre una diferencia social cualquiera y lo que socialmente se considera una desigualdad social.

Más aun, estudiar las formas de significación y construcción de sentido social de modo articulado con una visión sociológica o estructural, implica a su vez entender que estos procesos socioculturales no se realizan de manera aleatoria e individual sino que refieren a estructuras, posiciones, relaciones y valores que, por más diversos que resulten, se construyen siempre de manera colectiva, en procesos socio-históricos concretos.

Sin embargo, estos importantes avances, que permitirían un alto grado de sutileza y sofisticación de los análisis, no son en general difundidos y adoptados por otras disciplinas sociales -y menos aún por el "sentido común"- por lo que siguen muy vigentes las visiones esencialistas, etnocéntricas y estáticas de "la cultura" y las variables socioculturales que intervienen en los procesos sociales.

\subsection{Psicología y sociología. Dualismo entre individuo y sociedad}

Junto a la dualidad entre igualdad y diferencia se plantea desde el comienzo otra que atraviesa al pensamiento social: la tensión o dualidad entre individuo y sociedad. Esta se expresa también como el problema de la relación entre el sistema social y los comportamientos individuales, entre los colectivos sociales y las formas de subjetividad, o bien entre las determinaciones de la estructura social y la capacidad de agencia de los sujetos, u otras formulaciones similares. Ello resulta en gran parte resultado de las dificultades para un abordaje articulado al interior de las disciplinas sociales y de éstas con otras ciencias. Tal problema se presenta como una especie de abismo conceptual, por ejemplo, entre la psicología y la sociología.

Para avanzar en esta cuestión, nos interesa rescatar los aportes del sociólogo Norbert Elias, quien al respecto señala:

No es habitual, en un libro que trata de los problemas de la sociología, ocuparse con cierta extensión de la imagen que se tiene del individuo, de la persona individual. La especialización científica es actualmente tan rigurosa que si en la relación con los 
universales de la sociedad se incluyen en la reflexión no sólo problemas de los hombres en plural, sino también problemas de los hombres en singular, se da la sensación de proceder casi a un paso ilegal de frontera o tal vez incluso de proceder a un desplazamiento ilegítimo de los mojones que señalan la línea fronteriza (Elías, 1982, pp. 154 y 155).

Elías plantea que la separación convencional entre la investigación científica del hombre y de los hombres es problemática y constituye "un error intelectual" (ibídem), y afirma que la limitación disciplinar, no por motivos "objetivos" sino de "especialización" entre los campos de estudio, redunda en un empobrecimiento y errores conceptuales para ambos.

Como hemos señalado, sucede que, aún cuando se intenta un análisis más integrado y complejo de la realidad social, se lo hace desde nociones que no se condicen con los avances realizados por cada ámbito de especialidad, ya que constituyen aproximaciones unilaterales desde una u otra disciplina y no desde enfoques y metodologías interdisciplinarias, en términos de Rolando García (2007).

Por ejemplo, desde Sigmund Freud y los posteriores avances del psicoanálisis, sabemos que el hombre no constituye una unidad coherente e integrada, ya que una parte importante de su estructura psíquica permanece inconsciente. Ésta se vincula a través de complejos mecanismos y procesos con su parte consiente (el "yo") y con los ideales propuestos por su entorno familiar y social (el "súper yo" o "ideal del yo"). Sin embargo, poco se ha avanzado en las profundas implicancias que semejante descubrimiento tiene para el conjunto de las ciencias sociales. Por citar un ejemplo, la teoría de la acción racional -a que se echa mano desde la ciencia económica para explicar el comportamiento de los individuos- desconoce por completo esta dimensión constitutiva del hombre, incurriendo en errores conceptuales característicos del pensamiento racionalista. Del mismo modo, como señala Elias, la sociología convencional intenta clarificar la imagen de los hombres como sociedades pero no como individuos, con lo cual incurre en una visión del hombre "no crítica" o "pre-científica", es decir, abstracta o idealista.

Por otra parte, desde la psicología, e incluso desde la psicología social, cuando se pone al hombre individual en el centro de atención, dejando en los márgenes su inserción en la sociedad, o cuando ésta es un mero "medio" o "entorno", se pierde la visión de la totalidad y se reducen los problemas específicamente sociológicos y politológicos (como la configuración de la estructura social y el reparto del poder) a problemas psicológicos.

En un trabajo anterior (Altschuler, 2008) planteamos que los enfoques y aportes de la sociología y la psicología, desde las contribuciones de Norbert Elías y Sigmund Freud, parecían por momentos divergentes y por momentos similares pero en diversos planos (la sociedad y el individuo respectivamente) y, por tanto, resultaban complementarios. Planteamos así que los análisis de cada disciplina entraban en un terreno oscuro, justo allí donde empezaban los aportes de la otra, y calificamos a esta distancia (no real sino por motivos de especialización) 
como un abismo o hiato entre psicoanálisis y ciencias sociales ${ }^{5}$. Como profundizaremos luego, los aportes de Elías resultan fundamentales en este sentido, cuando afirma:

Lo que se caracteriza con dos conceptos distintos como individuo y sociedad no son, como el uso actual de estos conceptos a menudo hace aparecer, dos objetos que existan separadamente, sino dos planos distintos, pero inseparables, del universo humano (Elías, 1982, p. 156).

Retomando la problemática específica del poder y la desigualdad social, podemos preguntarnos, como lo hace Elías (1976) ¿Qué factores llevan a los hombres a diferenciarse, constituir relaciones de poder y de desigualdad? ¿Cómo se desarrollan estos procesos y por qué? ¿Qué hay por detrás de las relaciones de poder y desigualdad, más allá del interés por el beneficio económico o material? Este autor explica que el gran descubrimiento de Marx en relación a la búsqueda de ventajas económicas en las luchas sociales fue en verdad iluminador, pero a tal punto que encegueció otras miradas y oscureció otras necesidades que poseen los hombres y por las que luchan en sus relaciones, como la necesidad de auto-estima, reconocimiento $y$ valoración ${ }^{6}$. De este modo, Elias articula problemáticas sociológicas, económicas y políticas fundamentales (el poder, los privilegios económicos, la estructura y la desigualdad social) con dimensiones subjetivas, emotivas y hasta biológicas de los individuos, tendiendo así importantes puentes entre las disciplinas sociales, pero sin escindir además las dimensiones biológicas y físicas que atañen a la complejidad de la vida humana (Morin, 1994) ${ }^{7}$.

\section{Hacia un enfoque complejo de las desigualdades sociales}

\subsection{Algunos enfoques y conceptos para un abordaje interdisciplinario de la desigualdad social}

Como señala Morín (1977, p. 25), un enfoque complejo y multidimensional no implica un enciclopedismo que resultaría desalentador. De lo que se trata más bien es de encontrar puentes analíticos y conceptos clave que nos permitan articular los saberes especializados, no para negarlos sino, por el contrario, para integrarlos de una manera no disyuntiva ni reduccionista.

En este marco, un concepto que nos resulta de gran interés para avanzar en un camino de articulación interdisciplinaria y que además resulta muy significativo para el estudio de las desigualdades sociales es el concepto de figuración social de Norbert Elías, también conocido

\footnotetext{
${ }^{5}$ Dicho terreno oscuro sería para la psicología la sociedad, en tanto realidad compleja estructurada; y para la sociología las motivaciones y factores inconscientes que llevan a los individuos a identificarse o diferenciare.

"En su "Ensayo teórico de la relación entre establecidos y marginados" (1976), Elías interpela a las ciencias sociales, obnubiladas por enfoques racionalistas y economicistas.

${ }^{7}$ Elías aborda estas cuestiones en su monumental obra El proceso de la civilización. Investigaciones sociogenéticas y psicogenéticas (1989), en la que desarrolla con un detalle socio histórico considerable los mecanismos de coacción social y autocoacción que sobre las funciones biológicas impone la "civilización".
} 
como configuración social. Una configuración es entendida como el "entramado de acciones de un grupo de individuos interdependientes", y puede aplicarse tanto a grupos pequeños (una familia, un barrio, o un juego de naipes) como a sociedades integradas por miles o millones de individuos interdependientes, conformando "figuraciones complejas" y "cadenas de interdependencia" (Elías, 1982). Este concepto nos permite pensar al mismo tiempo la dimensión individual y la social, ya que hace eje en un enfoque relacional y sistémico de la sociedad, en tanto "red de relaciones interdependientes" (Ibídem). Si bien la relación individuosociedad constituye un tema clásico de la sociología, lo novedoso del planteo de Elías, es que dicha interrelación no se produce entre hombres abstractos, sino entre hombres concretos, es decir en toda su totalidad: "no sólo en su intelecto, sino con toda su persona, con todo su hacer y todas sus omisiones en sus relaciones recíprocas" (1982, p. 157). De este modo, se abre el camino para la integración de trayectorias de estudios recientes, como la sociología y antropología de las emociones y el cuerpo (Bericat Alastuey, 2000; Le Breton, 2012).

Desde este enfoque, Elías ubica en el centro de la cuestión el tema de la desigualdad y el poder, ya que la interdependencia de los individuos -premisa para que se constituya una figuración específica- siempre implica diferenciales de poder y valoración social. Así, no se trata sólo de una interdependencia de los hombres en tanto aliados, sino también como adversarios. En una figuración puede existir una jerarquía de varias relaciones "yo" y "el" o "nosotros" y "ellos", lo cual posee además toda una historicidad. En las cambiantes figuraciones históricas de poder, se producen "oscilaciones en el balance de poder" entre grupos sociales, conformando "equilibrios fluctuantes de poder", los cuales se cuentan entre las peculiaridades estructurales de todo proceso de figuración (Elias, 1976).

En resumen, el concepto de figuración social -simple y complejo al mismo tiempo- nos permite, a la vez, analizar de manera conjunta al individuo y su entramado social, y visualizar las relaciones de interdependencia, no sólo en función de la integración social sino también de los procesos de diferenciación, conflicto y desigualdad social.

En este camino y en post de estudios más articulados e interdisciplinarios para el abordaje de realidades complejas, queremos destacar también otros conceptos y enfoques que nos parecen significativos. Entre ellos, el concepto de hegemonía de Raymond Williams (1977). Tomando las concepciones de Gramsci -quien entiende a la hegemonía como una combinación entre consenso y coerción- Williams sostiene que la misma consiste en el establecimiento de límites y presiones a las prácticas y la imaginación social, en un determinado contexto sociohistórico. Para este autor, la hegemonía, alude al "complejo entrelazamiento de fuerzas políticas, sociales y culturales" que intervienen en la construcción de un orden social e instituyen la legitimidad de ciertas relaciones de dominación- subordinación (Williams, 1979: 129), constituyendo así un concepto clave para analizar la legitimidad de cierto orden de desigualdades sociales en un contexto socio-histórico dado. 
También, los conceptos de "estructuras del sentir" y "difusividad de las áreas de la vida social", del mismo autor (ibíd.) abonan a la comprensión de los sujetos y los procesos sociales en su trama compleja, multidimensional e interrelacionada. Otros enfoques que resultan alentadores en este sentido son los estudios sociales de la economía (que combinan miradas sociológicas y antropológicas sobre los fenómenos económicos) y la ya mencionada sociología y antropología de las emociones, en tanto se propone (re) introducir y poner en el centro del análisis de los procesos y estructuras sociales las cuestiones subjetivas, emocionales y vinculares.

Son de destacar también importantes abordajes contemporáneos que proponen pensar las desigualdades sociales como fenómenos complejos, multidimensionales y multideterminados. Los aportes del antropólogo mexicano Luis Reygadas son significativos en este sentido. En su libro La apropiación. Destejiendo las redes de la desigualdad (2008), Reygadas retoma los aportes de Weber y Marx -a quienes considera "los pilares" de las teorías actuales sobre la desigualdad-, señalando que ambos pensadores hacen hincapié en la explicación de las desigualdades en el marco de relaciones de poder. Reygadas plantea además una visión relacional, procesual y disputada -en cuanto a su legitimidad- del fenómeno de la desigualdad. Entiende a ésta de modo multidimensional (en el reconocimiento de distintos tipos de desigualdades, incluyendo no sólo los aspectos económicos sino también los políticos y simbólicos) y multideterminada (ya que intervienen de forma articulada los niveles individuales, relacionales y estructurales).

También resultan relevantes los trabajos de Charles Tilly quien, en su análisis de la "desigualdad persistente" (Tilly, 2000) discute abiertamente con el "individualismo metodológico", el cual tiende a explicar la desigualdad como resultado de procesos mentales, decisiones y aptitudes individuales o grupales. En línea con el enfoque de Reygadas, Tilly aboga por un enfoque relacional, que pone el acento en los lazos sociales, "en los vínculos y no en las esencias" y en perspectiva histórica, a fin de comprender las acumulaciones desiguales que producen efectos en el presente (por ejemplo la falta de acceso a una alimentación y educación de calidad).

Dichos avances vienen a plantear que el reconocimiento de las múltiples dimensiones de la desigualdad, si bien constituye un avance, no resulta suficiente. Es necesario además analizar en cada configuración social particular, cómo se entrelazan estas desigualdades y los resultantes de su efectos combinados. Así, es de interés analizar en contextos históricos y culturales concretos, las implicancias de ocupar ciertas posiciones sociales, por ejemplo, ser mujer, negra y pobre; o ser indígena y vivir en regiones aisladas y ambientalmente degradadas, con escaso acceso a recursos y participación política ${ }^{8}$.

\footnotetext{
${ }^{8}$ Un grupo de investigadores de Alemania y América Latina se encuentra desarrollando esta perspectiva de "desigualdades entrelazadas e interdependientes" en los últimos años, a través de la red desiguALdades.net. Tuve oportunidad de participar de la misma en ocasión de la "Escuela de
} 
Creemos necesario considerar también cómo interactúan las diversas escalas (lo local, regional, nacional y global) en la producción de procesos y fenómenos sociales particulares y en el entrelazamiento de las desigualdades en escenarios concretos, ya que los recortes meramente locales y/o nacionales no dan cuenta de tal compleja interacción.

Por último, pero no por ello menos importante, resulta clave orientar los estudios no sólo hacia la comprensión de los mecanismos de producción de las desigualdades sociales, sino también los de reproducción, legitimación y cuestionamientos de las mismas (Grimson, et. al, 2008). Ello requiere analizar los mecanismos materiales y simbólicos que se ponen en juego en las interacciones cotidianas que actualizan y/o disputan las desigualdades sociales, es decir, los mecanismos de construcción de hegemonía y legitimidad de un orden social, en su carácter dinámico y disputado. Estos nos permiten analizar en qué medida las desigualdades sociales son "naturalizadas" y por tanto "invisibilizadas", o por el contrario son reconocidas y/o cuestionadas como "injustas" por los sujetos sociales en cada momento histórico y contexto socio-cultural. $^{9}$

\subsection{Aportes del paradigma de la complejidad}

En el recorrido realizado hasta aquí, hemos puesto a jugar diversos conceptos y enfoques que se relacionan con el paradigma de la complejidad, y que consideramos nos permiten abordar algunas de las dificultades de las ciencias sociales señaladas, en función de producir estudios más integrados de las desigualdades sociales. Sistematizamos ahora tales contribuciones y realizamos puentes analíticos con los enfoques propuestos.

El paradigma complejo nos brinda una herramienta epistemológica fundamental para reflexionar críticamente sobre los obstáculos y limitaciones de las ciencias sociales en su devenir y en la actualidad. Nos referimos ya a la identificación de algunos dualismos que las atraviesan y que entendemos como resultado del pensamiento disyuntivo, reduccionista y simplificador a que alude Morin (1977 y 1994), y a la especialización desarticulada en que se desarrollaron históricamente las ciencias.

Este paradigma aporta elementos para superar estos dualismos y aproximarnos a un pensamiento dialéctico de la realidad, ya que "la aceptación de la complejidad es la aceptación de una contradicción" (Morin, 1994, p. 95). También, para el reconocimiento de la interrelación o dependencia mutua de las dimensiones que habían sido separadas o aisladas (lo socioantropológico, lo biológico y lo físico), pero no en la forma de un "círculo vicioso" sino de un espiral enriquecedor. En palabras de Morin:

\footnotetext{
Verano sobre desigualdades interdependientes en América Latina", realizada en Sao Pablo, Brasil, del 1 al 5 de noviembre de 2010.

${ }^{9}$ Esta línea de estudios es desarrollada por el proyecto de investigación "Procesos de legitimación de la desigualdad social en la Argentina actual" (UNSAM- PICT), con sede en el Instituto de Altos Estudios Sociales (IDAES), del cual formé parte como becaria doctoral (2008-2011).
} 
... Siempre se han roto los círculos viciosos, ya sea aislando las proposiciones, ya sea eligiendo uno de los términos como principio simple al cual deben reducirse los demás [...] Entrevemos la posibilidad de transformar los círculos viciosos en ciclos virtuosos, que lleguen a ser reflexivos y generadores de un pensamiento complejo. De ahí esta idea que guiará nuestra partida: no hay que romper nuestras circularidades, por el contrario, hay que velar para no apartarse de ellas. El círculo será nuestra rueda, nuestra ruta será el espiral (Morin, 1977, pp. 31 y 32).

De este modo, el enfoque de la complejidad propone un método de conocimiento que permite distinguir sin desarticular y asociar sin identificar ni reducir. Ello hace posible repensar los dualismos de las ciencias sociales que identificamos en este trabajo: individuo/ sociedad e igualdad/ diferencia. Respecto del primero señala Morin:

Es, pues, de primera necesidad, no sólo rearticular individuo y sociedad (cosa que comenzó en ocasiones, aunque al precio del aplastamiento de una de las dos nociones en provecho de la otra), sino también efectuar la articulación reputada de imposible (peor, de "superada") entre la esfera biológica y la esfera antropo-social (Morin, 1977, p. 22).

En cuanto al dualismo igualdad/ diferencia, la complejidad implica también la rearticulación de lo complementario y lo antagónico, de lo uno y lo diverso (como bien lo entendía Marx y como lo propone Elías con su concepto de figuración), es decir, la conceptualización de lo concreto como la unidad de la diversidad (Morin, 1977 y 1944, García 2007) ${ }^{10}$.

Ahora bien, ¿cómo avanzar hacia investigaciones multidimensionales y enfoques complejos? Los aportes de Rolando García resultan fundamentales en este sentido. A partir de su Teoría de los sistemas complejos, este autor se propone brindar una metodología de trabajo interdisciplinario para abordar tales sistemas, así como un marco conceptual que lo fundamenta (García, 2007, p. 39). La investigación interdisciplinaria supone la conformación de un equipo multidisciplinar que, a partir de la interacción entre sus miembros, define en forma conjunta la problemática de estudio, implicando además la elaboración de un marco conceptual común y de prácticas de investigación convergentes (2007, pp. 33 y 67). Se produce en este proceso una tensión permanente entre especialización disciplinar e interdisciplina, ya que se trabaja también aquí con la unidad en la diversidad. Esto se da en un doble proceso que García denomina diferenciación de una totalidad y reintegración conceptual enriquecida, a partir de los hallazgos disciplinares específicos y su interrelación (2007, p. 68). En palabras de García: "Los objetivos de una investigación interdisciplinaria se logran a través del juego dialéctico en las fases de diferenciación e integración que tienen lugar en el proceso de definición y estudio de un sistema complejo" (2007, p. 101). Habría que considerar asimismo en dicho proceso la intervención e

\footnotetext{
${ }^{10}$ De todos modos, como advierte Morin, reconocer el carácter multidimensional de toda realidad, es decir, su complejidad, no es aspirar a la completud o totalidad del conocimiento, ya que hay que aceptar que no podemos escapar nunca a la incertidumbre y la "incompletud" (Morín, 1994, p. 101). Es quizás éste uno de los aportes más originales del paradigma en relación a enfoques previos sobre la totalidad, los sistemas, el holismo, etc.
} 
incidencia de relaciones de poder y construcciones hegemónicas que imponen ciertas jerarquías disciplinares y miradas dominantes en contextos socio-históricos específicos, dando lugar a disputas, conflictos y tensiones intra e interdisciplinares.

Otro elemento trabajado por García que resulta clave se vincula al problema del "recorte" de la problemática de estudio desde una perspectiva compleja, ya que dadas sus múltiples dimensiones e interrelaciones su estudio parecería inabarcable. Al respecto, sus principales aportes refieren a la construcción de un sistema complejo, en tanto "trozo de la realidad" que incluye aspectos físicos, biológicos, sociales, económicos y políticos (Ibíd., p. 47), cuyos límites no están definidos, pero pueden definirse. El recorte implica tener muy en cuenta las interacciones entre el "afuera" y el "adentro" del sistema definido, así como las escalas espaciales y temporales que tomamos para su definición. Al respecto resulta fundamental la consideración de que "dejar afuera de los límites del sistema no significa necesariamente dejar fuera de consideración" (2007, p. 49). Así García propone el concepto de condiciones de contorno o condiciones en los límites, es decir, el análisis de los diversos flujos que interactúan con el sistema.

Asimismo, queremos rescatar el concepto de "interdefinibilidad", el cual implica que los componentes de un sistema no son independientes sino que se determinan y definen mutuamente (ibídem). Es interesante rescatar la convergencia de este enfoque con el concepto de figuración, en cuanto a su dimensión relacional y estructural. Veamos:

La elección de los límites [de un sistema] debe realizarse en forma tal que aquello que se va a estudiar presente cierta forma de organización o estructura. Como la estructura está determinada, a su vez, por el conjunto de las relaciones, está claro que el sistema debe incluir aquellos elementos entre los cuales se han podido detectar las relaciones más significativas. Los otros elementos quedan "afuera". Las interrelaciones entre ellos y los elementos que quedan dentro determinan las condiciones de los límites (García, 2007, p. 49).

Por último, nos parece importante rescatar el señalamiento tanto de Morín como de García en su crítica epistemológica al paradigma científico dominante, sobre la necesidad de visibilizar y contextuar al "observador" o "analista". Éste no constituye nunca un "mero observador" o un individuo "neutral". Por el contrario, el investigador es siempre un sujeto situado, histórica, espacial y culturalmente, por lo que estos elementos deben reintroducirse en el análisis para dar cuenta del tipo de conocimiento o datos producidos. Esta perspectiva se encuentra en línea claramente con las perspectivas antropológicas actuales que, en base a los aportes de la etnometodología de Harold Garfinkel de mediados del siglo XX, plantean esta problemática desde el concepto de "reflexividad" y conciben al trabajo de campo -ya sea a través de entrevistas o de la etnografía- como una relación social, en la cual el investigador es parte del tipo de conocimiento que produce, y por tanto debe considerar esta dimensión en su trabajo (Althabe y Hernández, 2005; Hernández y Svampa, 2008; Guber, 2001). Si bien esto es 
pertinente para cualquier problemática social, lo es particularmente para abordar la cuestión de la desigualdad social.

\section{Conclusiones}

Hemos intentado mostrar que la desigualdad social constituye un problema teórico complejo y que su estudio implica importantes desafíos. Es complejo en la medida en que resulta un fenómeno multidimensional y multideterminado, cuya comprensión requiere el análisis de su devenir histórico, contextual, situado, involucrando asimismo escalas temporales, sociopolíticas y espaciales diversas e interrelacionadas. También porque se encuentra mediado culturalmente y fuertemente atravesado por relaciones de poder que, además, nos involucran directamente como investigadores y como actores sociales.

Hemos señalado también que, particularmente desde los años 70 y 80 , se produjeron avances significativos en las diversas disciplinas sociales hacia enfoques más complejos e integrados. Identificamos algunos conceptos y enfoques que consideramos clave para avanzar en este sentido -especialmente en la obra de Norbert Elías, pero también de Raymond Williams y, más recientemente de Luis Reygadas y Charles Tilly-, y por último establecimos algunos puentes y convergencias de estos con el paradigma de la complejidad. Éstas se fundan en el peso puesto en los análisis relacionales, de entramados y redes de interdependencia, que no dejan de lado la historicidad, el poder y la constitución de las subjetividades, sino que, por el contrario, trabajan en base a estas cuestiones. De este modo, permiten la articulación -y no la disyunción- entre dimensiones de la vida humana, posibilitando pensar al mismo tiempo lo complementario y lo antagónico, lo uno y lo diverso.

Hemos sostenido que tales avances en las ciencias sociales no constituyen modos generalizados ni sistemáticos de reflexión e investigación y menos aún que se haya avanzado lo suficiente en herramientas concretas de trabajo interdisciplinario que alcancen las prácticas cotidianas de investigación. Estos avances resultan actualmente, más o menos significativos, al interior de las ciencias sociales, mientras que, tal como lo afirma Morin, nos encontramos lejos de pensar las articulaciones de estos conocimientos socio- antropológicos con las dimensiones físicas y biológicas que componen la complejidad de la vida humana. Al respecto, y habiéndome formado al interior de las ciencias sociales ${ }^{11}$, considero que existen fuertes resistencias desde las mismas para realizar estas articulaciones del pensamiento. Ello se explica a mi entender por la fuerte impronta que tuvieron, especialmente en su origen, los enfoques reduccionistas de la realidad social, ligados a la hegemonía de las "ciencias naturales" y que se tradujeron en enfoques organiscistas y biologiscistas y en una asimilación general de los métodos de investigación de estas ciencias. Tales "fantasmas" obstaculizan aún hoy una apertura hacia una

\footnotetext{
${ }^{11}$ Primero como Socióloga y luego en el Doctorado en Ciencias Sociales (UNGS- IDES).
} 
articulación no reduccionista sino enriquecedora de la realidad social, tal como lo plantean García y Morin.

De todos modos, existen algunas aperturas importantes de lo social hacia las dimensiones físicas y biológicas. Considero que los trabajos de Elías son un puente fundamental al respecto y que el estudio de las desigualdades sociales constituye una oportunidad en este sentido. Ya a modo de interrogantes e hipótesis para futuras investigaciones, podemos preguntarnos si la "igualdad" -a partir de la cual pensamos las desigualdades y que, por otra parte, resulta un horizonte o ideal no absoluto- se vincula de algún modo a cierto sustrato humano, en tanto especie: a pesar de las jerarquías y posiciones diferenciadas en la vida social, todos tenemos, cada día, hambre, cansancio, deseos o necesidades de diverso tipo. Esta dimensión biológica, que se vincula a la relación del hombre con la naturaleza, escisión fundamental que estamos lejos de rearticular, es lo que de algún modo nos "iguala" e incluso desde ese horizonte, nos permite pensar y hablar de "desigualdad".

De este modo, coincidimos con Morin en que la complejidad no es un "concepto solución" que viene a cerrar dilemas abiertos, sino que su desafío es la posibilidad de "pensar complejamente como metodología de acción cotidiana" (Morin, 1994), en un camino que se va haciendo al andar.

\section{Referencias Bibliográficas}

Althabe G. y Hernández V. (2005). Implicación y reflexividad. En Hernández, et al. (comps), Etnografias Globalizadas. Buenos Aires: Ediciones SAA.

Altschuler, B. (2016). "Desigualdades y Fronteras Sociales en la Configuración de la Vitivinicultura Mendocina". Tesis de Doctorado en Ciencias Sociales UNGS- IDES, Buenos Aires (inédito).

Altschuler, B. (2014). Disputas y fronteras sociales en la configuración actual de la vitivinicultura mendocina. Aportes de la sociología figuracional de Norbert Elías. En revista Prácticas de Oficio. Investigación y reflexión en Ciencias Sociales, 14. Buenos Aires: IDES. Recuperable en: http://ides.org.ar/wp-content/uploads/2015/02/Altschuler-texto2.pdf

Altschuler, B. (2008). "Freud y las ciencias sociales. Pensando el lazo social en las relaciones entre establecidos y marginados de Norbert Elias", Doctorado IDES- UNGS, (Inédito).

Altschuler, B. (2008). El pensamiento de Raymond Williams en el análisis de la Economía Social. Revista Prácticas de Oficio. Investigación y Reflexión en Ciencias Sociales, 3. Buenos Aires: IDES. Recuperable en: http://ides.org.ar/wpcontent/uploads/2012/04/artic111.pdf

Barth, F. (1976). Los grupos étnicos y sus fronteras. México: FCE. 
Bericat Alastuey, E. (2000). La sociología de la emoción y la emoción en la sociología. Revista Papers 62, pp. 145 a 167. España: Universidad de Málaga.

Bourdieu, P. (1984). Espacio social y génesis de las 'clases'. En Bourdieu, P.: Sociología y cultura. México: Grijalbo.

Bourdieu, P. (1979). La distinción. Criterio y bases sociales del gusto. Madrid: Taurus.

Brubaker, R. y Cooper, F. (2002). Más allá de identidad. En Apuntes de investigación 7, Buenos Aires.

Castel, R. (1995 [1977]). La metamorfosis de la cuestión social. Una crónica del salariado, Buenos Aires: Paidós.

Cuche, D. (1996). La noción de cultura en las ciencias sociales. Buenos Aires: Nueva Visión.

De Ipola, E. (2005). Intervención De Ipola. Seminario Internacional en Psicoanálisis y Ciencias Sociales. Argentina: FLACSO.

Durkheim, E. (1895). Las reglas del método sociológico. Buenos Aires: Prometeo.

Elias, N. (1976). Ensayo Teórico sobre las Relaciones entre Establecidos y Marginados. En Elias, N.: La Civilización de los Padres y Otros Ensayos. Bogotá: Norma.

Elias, N. (1982). Sociología Fundamental. Barcelona: Gedisa.

Elias, N. (1998). El proceso de la civilización. Investigaciones sociogenéticas y psicogenéticas. México: Fondo de Cultura Económica.

Freud, S. (1921). Psicología de las masas y análisis del Yo. En Obras Completas. Argentina: Amorrortu.

Freud, S. (1930). Malestar en la cultura. En Obras Completas. Argentina: Amorrortu.

García, R. (2007). Sistemas complejos. Conceptos, método y fundamentación epistemológica de la investigación interdisciplinaria. Barcelona: Gedisa.

Giddens, A. (1996 [1979]). La estructura de clases en las sociedades avanzadas. Madrid: Alianza.

Grimson, A. y Semán, P. (2005). Presentación: la cuestión cultura. En Etnografías contemporáneas, 1, (1), pp. 11-22.

Grimson, A. et. al. (2008). Naturalización y legitimación de las desigualdades sociales en la Argentina, UNSAM-PICT (proyecto de investigación, inédito).

Grimson, A. (2008). "Legitimación de la desigualdad social". En Congreso de la Latin American Studies Association (LASA), Río de Janeiro.

Guber, R. (2001). Método, campo y reflexividad. Buenos Aires: Norma.

Hernández V. y Svampa M. (2008). Entre varios mundos: Reflexividad, conocimiento y compromiso. Buenos Aires: Prometeo. 
Kessler, G. (2014). Controversias sobre la desigualdad. Argentina, 2003- 2013. Buenos Aires: Fondo de Cultura Económica de Argentina.

Laclau, E. (2005). La razón populista. México: Fondo de Cultura Económica.

Lamont, M. y Molnár, V. (2002). The Study of boundaries in the Social Sciences. En Annual Review of Sociology, 28.

Le Breton, D. (2012). Por una antropología de las emociones. En Revista Latinoamericana de Estudios sobre Cuerpos, Emociones y Sociedad 10.

Morin, E. (1994). Introducción al pensamiento complejo. Barcelona: Gedisa.

Morin, E. (1977). El Método. Tomo I: La naturaleza de la naturaleza. Multiversidad Mundo Real. Sitio Web oficial de Edgar Morin, Ediciones especiales.

Morin, E. (1984). Ciencia con conciencia. Barcelona: Anthropos.

Ortner, S. (2005). Geertz, subjetividad y conciencia posmoderna. En Etnografías contemporáneas. 1 (1), pp. 25-54.

Reygadas, L. (2008). La apropiación. Destejiendo las redes de la desigualdad. Barcelona: Antropos.

Rosanvalon, P. (1995). La nueva cuestión social. Repensar el Estado Providencia. Buenos Aires: Manantial.

Schorske, C. (1981). Viena fin de siglo. Política y cultura. Barcelona: Gustavo Gili.

Svampa, M. (2005). La sociedad excluyente. La Argentina bajo el signo del neoliberalismo. Buenos Aires: Taurus.

Tilly, C. (2002). La desigualdad persistente. Buenos Aires: Manantial.

Wieviorka, M. (2006). Cultura, Sociedad y Democracia. En Gutiérrez Martínez, D., Multiculturalismos: Perspectivas y Desafíos. México: El Colegio de México y Siglo XXI.

Williams, R. (1977). Marxismo y Literatura. Barcelona: Península. 